\title{
Electroweak diboson production at ATLAS
}

\author{
A. Solodkov on behalf of the ATLAS Collaboration \\ State Research Center of Russian Federation - Institute for High Energy Physics of National \\ Research Center "Kurchatov Institute" - 1, Nauki square, 142281, Protvino, Moscow region, \\ Russia
}

received 26 July 2016

\begin{abstract}
Summary. - This paper is giving an overview of recent ATLAS results on the production cross sections of gauge boson pairs decaying leptonically using data from pp collisions at $\sqrt{s}=13 \mathrm{TeV}$ for $Z Z$ and at $\sqrt{s}=8 \mathrm{TeV}$ for $W^{ \pm} Z$ and $W^{ \pm} W^{ \pm}$ at the LHC at CERN. The cross sections are found to be in agreement with the expectations from the Standard Model within the estimated uncertainties. The production cross section measurements also allow for studies of anomalous triple and quartic gauge couplings for which $95 \%$ confidence level limits are set.
\end{abstract}

\section{1. - Introduction}

The measurement of the production of pairs of electroweak gauge bosons plays a central role in tests of the Standard Model (SM) and in searches for new physics at the TeV scale. In the SM, Triple Gauge Couplings (TGC) at tree level are predicted only when there are charged bosons involved, while vertices with three neutral bosons are forbidden. The TGC vertex is completely fixed by the electroweak gauge structure and therefore a precise measurement of this vertex, through the analysis of diboson production, is essential to test the high energy behavior of electroweak interactions and to probe for possible new physics in the bosonic sector. The non-Abelian gauge nature of the SM predicts, in addition to the TGCs, quartic gauge boson couplings (QGC). Any deviation from gauge constraints can cause a significant enhancement in the production cross section at high diboson invariant mass due to anomalous triple and quartic gauge boson couplings (aTGC and aQGC).

This note presents measurements of the diboson production cross sections in protonproton collisions and limits on aTGC and aQGC with the ATLAS experiment [1] at the LHC. The following diboson pair final states were investigated: $W^{ \pm} Z, W^{ \pm} W^{ \pm}$at center-of-mass energy $\sqrt{s}=8 \mathrm{TeV}$ and $Z Z$ at $\sqrt{s}=13 \mathrm{TeV}$. For $Z Z$ pairs a sample of integrated luminosity $L=3.2 \mathrm{fb}^{-1}$ of 2015 data was used and for $W^{ \pm} Z, W^{ \pm} W^{ \pm}$ analyses the data collected in 2012 correspond to $L=20.3 \mathrm{fb}^{-1}$. All studies used the fully leptonic decay final states with $Z \rightarrow l l$ and $W \rightarrow l \nu$, where $l=e, \mu$.

(C) CERN on behalf of the ATLAS Collaboration 

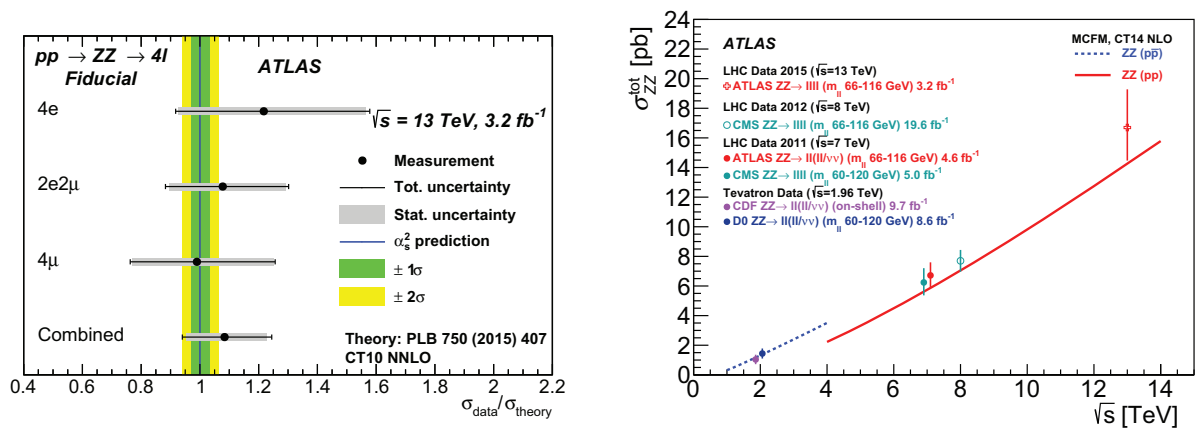

Fig. 1. - Left: Comparison between measured $Z Z$ fiducial cross sections and $\mathcal{O}\left(\alpha_{S}^{2}\right)$ predictions [2]. Right: Total $Z Z$ cross section compared to measurements at lower center-of-mass energies by ATLAS, CMS, CDF, and D0, and to a prediction from MCFM at $\mathcal{O}\left(\alpha_{S}^{1}\right)$ accuracy for the $q \bar{q}$-initiated process and at $\mathcal{O}\left(\alpha_{S}^{2}\right)$ accuracy for the loop-induced $g g$-initiated process [2]. A full $\mathcal{O}\left(\alpha_{S}^{2}\right)$ prediction (known to improve agreement at $\sqrt{s}=13 \mathrm{TeV}$ ) was not yet available for all the different center-of-mass energies. Some data points are shifted horizontally to improve readability.

\section{2. $-\mathrm{ZZ}$ cross section measurements}

The $\mathrm{Z}$ boson pair production at $\sqrt{s}=13 \mathrm{TeV}$ is measured in final states with 2 pairs of charged leptons [2]. The cross section is found by counting candidate events, subtracting the expected contribution from background events, correcting for detector effects, and dividing by the integrated luminosity. It is measured in a fiducial phase space that corresponds closely to the experimental acceptance. In addition, an extrapolation of the cross section to a total phase space for $Z$ bosons is performed. The presented cross section measurements are inclusive with respect to additional jets.

The $4 l$ state is a very clean signature with small background contributions. Background events from processes with at least four prompt leptons in the final state are estimated with the Monte Carlo (MC) samples. Contributions of $0.07 \pm 0.02$ events from $Z Z$ processes where at least one $Z$ boson decays to $\tau$ leptons, $0.17 \pm 0.05$ events from non-hadronic triboson processes, and $0.30 \pm 0.09$ events from all-leptonic $t \bar{t} Z$ processes are predicted. Events from processes with two or three prompt leptons, e.g. $Z, W W$, $W Z, t \bar{t}$ and $Z Z$ events with one $Z$ boson decaying hadronically, where associated jets or photons contain or fake a nonprompt lepton, can pass the event selection. This background contribution is estimated using data-driven methods and is found to be $0.09_{-0.04}^{+1.08}$ events. The total expected number of background events is $0.62_{-0.11}^{+1.08}$ events and number of events observed in data is 63 , of which 15,30 , and 18 are in the $4 e, 2 e 2 \mu$, and $4 \mu$ channel, respectively.

The measured fiducial cross sections are shown in table I and fig. 1(left) along with a comparison to $\mathcal{O}\left(\alpha_{S}^{2}\right)$ calculations. Table I also shows the total combined cross section. The CT10 NNLO PDFs and a dynamic scale equal to the mass of the four-lepton system are used in the calculation. Overall good agreement with the predictions from the SM is found. The dominant systematic uncertainties come from the uncertainties of the scale factors used to correct lepton reconstruction and identification efficiencies in the simulation and the choice of MC generator.

The measured total cross section is compared to measurements at lower center-of-mass energies and to a prediction from MCFM generator with the CT14 NLO PDFs, which is calculated at $\mathcal{O}\left(\alpha_{S}^{1}\right)$ accuracy for the $q \bar{q}$-initiated process and at $\mathcal{O}\left(\alpha_{S}^{2}\right)$ accuracy for the 
TABLE I. - Cross section measurement results for $Z Z$ pair production at $\sqrt{s}=13$ TeV compared to the $\mathcal{O}\left(\alpha_{S}^{2}\right)$ standard model predictions. The per-channel and combined fiducial cross sections are shown along with the combined total cross section.

\begin{tabular}{lcc}
\hline & Measurement & $\mathcal{O}\left(\alpha_{S}^{2}\right)$ \\
\hline$\sigma_{Z Z \rightarrow e^{+} e^{-} e^{+} e^{-}}^{\text {fid }}$ & $8.4_{-2.0}^{+2.4}(\text { stat })_{-0.2}^{+0.4}(\text { syst })_{-0.3}^{+0.5}($ lumi $) \mathrm{fb}$ & $6.9_{-0.2}^{+0.2} \mathrm{fb}$ \\
$\sigma_{Z Z \rightarrow e^{+} e^{-} \mu^{+} \mu^{-}}^{f i d}$ & $14.7_{-2.5}^{+2.9}(\text { stat })_{-0.4}^{+0.6}(\text { syst })_{-0.6}^{+0.9}($ lumi $) \mathrm{fb}$ & $13.6_{-0.4}^{+0.4} \mathrm{fb}$ \\
$\sigma_{Z Z \rightarrow \mu^{+} \mu^{-} \mu^{+} \mu^{-}}^{\text {fid }}$ & $6.8_{-1.5}^{+1.8}(\text { stat })_{-0.3}^{+0.3}(\text { syst })_{-0.3}^{+0.4}($ lumi $\mathrm{fb}$ & $6.9_{-0.2}^{+0.2} \mathrm{fb}$ \\
$\sigma_{Z Z \rightarrow l^{+} l^{-} l^{+} l^{-}}^{\text {fid }}$ & $29.7_{-3.6}^{+3.9}(\text { stat })_{-0.8}^{+1.0}(\text { syst })_{-1.3}^{+1.7}($ lumi $) \mathrm{fb}$ & $27.4_{-0.8}^{+0.9} \mathrm{fb}$ \\
$\sigma_{Z Z}^{\text {tot }}$ & $16.7_{-2.0}^{+2.2}(\text { stat })_{-0.7}^{+0.9}(\text { syst })_{-0.7}^{+1.0}($ lumi $) \mathrm{pb}$ & $15.6_{-0.4}^{+0.4} \mathrm{pb}$ \\
\hline
\end{tabular}

loop-induced $g g$-initiated process and is shown $v s$. center-of-mass energy in fig. 1(right). The cross section increases by a factor of more than two with a center-of-mass energy increase from $8 \mathrm{TeV}$ to $13 \mathrm{TeV}$.

\section{3. - WZ cross section measurements}

The analysis of $W Z$ boson pairs is performed in $3 l \nu$ final states [3]. The main sources of background come from $Z+$ jets and $t \bar{t}$ events, where the two leptons from the vector boson decays are accompanied by a jet which is misidentified as a lepton. These backgrounds are estimated using data-driven techniques. There is also a contribution from $Z Z$ events in which one of the leptons (especially muons) falls outside the acceptance of the detector and thus creates $E_{T}^{m i s s}$. This source is estimated from MC.

Ratios of fidutial cross section to the SM NLO predictions in different decay channels are shown in fig. 2(left). The measured cross section is $20 \%$ larger than SM prediction. However, the SM prediction, which is at NLO accuracy in perturbative QCD, is highly sensitive to the choice of renormalization scale $\mu_{R}$. In addition, new perturbative effects appearing at NNLO could enhance the SM prediction compared to the NLO calculation.
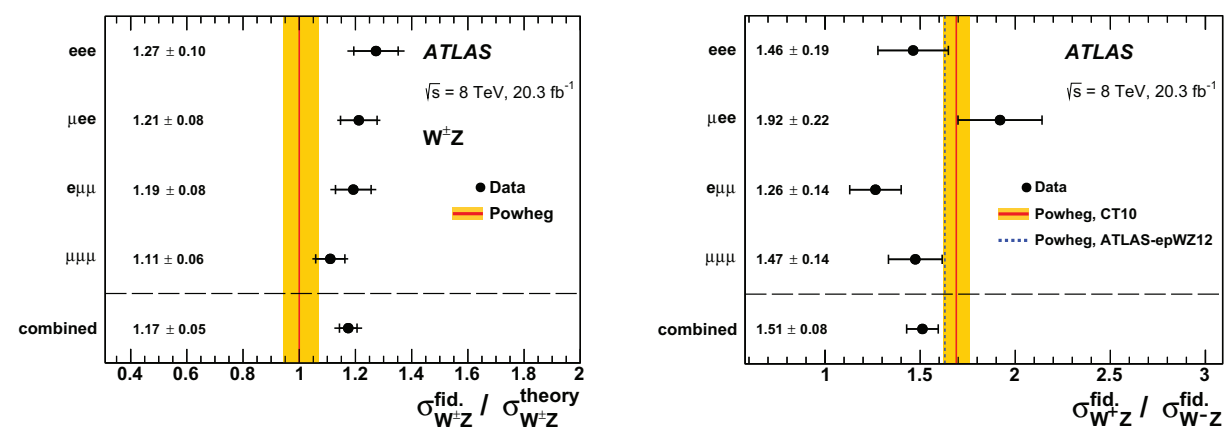

Fig. 2. - Left: Ratio of the measured $W^{ \pm} Z$ integrated cross sections in the fiducial phase space to the NLO SM prediction from POWHEG+PYTHIA using the CT10 PDF set [3]. The shaded orange band represents the uncertainty associated with the SM prediction. Right: Measured ratios $\sigma_{W+Z}^{f i d} / \sigma_{W-Z}^{f i d}$ of $W^{+} Z$ and $W^{-} Z$ integrated cross sections in the fiducial phase space in each of the four channels and for their combination [3]. The error bars on the data points represent the total uncertainties, dominated by statistical uncertainties. 
In fig. 2(right) ratio $W^{+} Z$ to $W^{-} Z$ production cross sections is compared with theoretical predictions, showing that such a ratio is sensitive to the choice of PDF set. Most of the systematic uncertainties cancel in the ratio and the measurement is dominated by the statistical uncertainty.

The $W^{ \pm} Z$ production cross section is measured as a function of several kinematic observables. Selected differential cross sections are presented in fig. 3. Among them cross section as a function of transverse momentum of the $Z$ boson, as a function of $p_{T}$ of the neutrino associated with the decay of the $W$ boson, as a function of the absolute difference between the rapidities of the $Z$ boson and the lepton from the decay of the $W$ boson, $\left|y_{Z}-y_{l, W}\right|$. The differential cross sections as a function of the transverse momenta of the neutrino from the $W$ decay is interesting because of its sensitivity to the polarization of the $W$ boson. The rapidity correlations between the $W$ and $Z$ decay products have been found to be useful tools in searching for the approximately zero $W Z$ helicity amplitudes expected at LO in the SM or for aTGC. These rapidity correlations are also sensitive to QCD corrections, PDF effects, and polarization effects of the $W$ and $Z$ bosons. Bottom right plot in fig. 3 shows the exclusive multiplicity of jets unfolded at particle level.

\section{4. - WW cross section measurements}

The $W W \rightarrow l \nu l \nu$ signal is measured in final states with two oppositely charged isolated leptons and large missing transverse energy [4]. Candidate $W W$ events are required to have no jets reconstructed in the final state because the background dominates at higher jet multiplicities as can be seen in fig. 4(left), where the reconstructed jet multiplicity is shown for data and MC.

Processes that can mimic the $l l+E_{T}^{m i s s}$ signal with no reconstructed jets are topquark production, when then event contain jets falling outside the acceptance and thus passing the jet veto requirement, $W+$ jets or multijet production processes where one or more jets are misidentified as leptons (fake leptons), Drell-Yan with mismeasured jets and diboson channels. Backgrounds stemming from top-quark, Drell-Yan, $W+$ jets and multijet production are evaluated using data-driven methods. The background from diboson production processes is modelled using MC samples normalized to the expected production cross section using theoretical calculations at the highest available order. Other processes, such as double parton interactions, vector-boson fusion processes or associated $W H$ production, resulting in $e \mu$, ee and $\mu \mu$ final states are not considered explicitly in the analysis as their contribution to the selected event sample is expected to be negligible $(<0.6 \%)$.

The total cross sections are measured separately in the three different final states and then combined. The combined cross section is found to be compatible with the NNLO prediction within about 1.4 standard deviations. A graphical comparison between the individual measurements and their combination is shown in fig. 4(right).

Two selected unfolded differential cross sections are shown in fig. 5. The predictions generally undershoot the data, except for high $p_{T}$ of the leading lepton (fig. 5(left)) and for high values of $\Delta \phi_{l l}$ (fig. 5 (right)), where there is a small deficit in data compared to the expectation from either MC prediction. In general, the shapes of the unfolded data distributions agree with either prediction at the level of $\pm 15 \%$. 

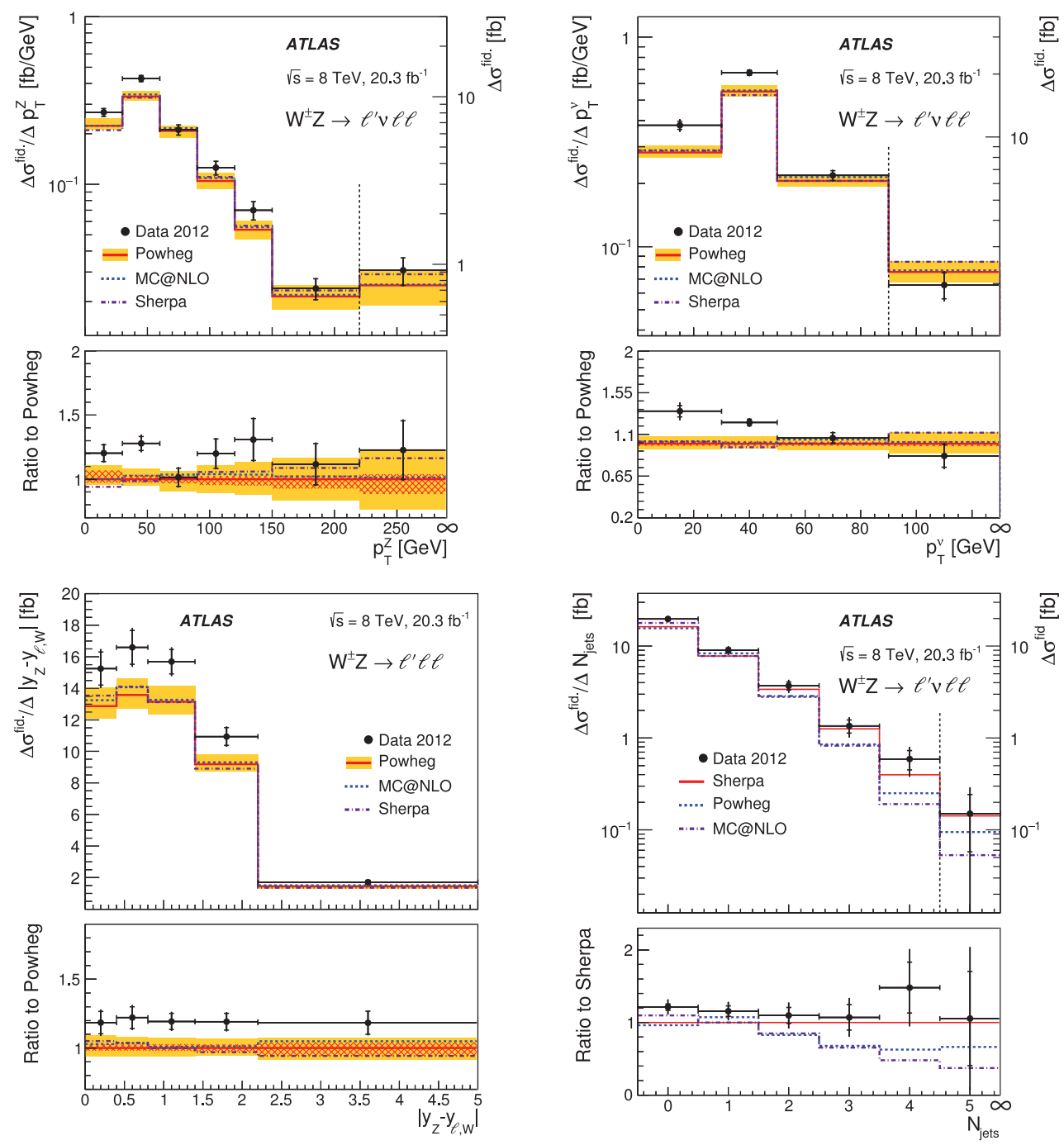

Fig. 3. - The measured $W^{ \pm} Z$ differential cross section in the fiducial phase space as a function of $p_{T}^{Z}$ (top left), $p_{T}^{\nu}$ (top right) and $\left|y_{Z}-y_{l, W}\right|$ (bottom left) [3]. The inner and outer error bars on the data points represent the statistical and total uncertainties, respectively. The measurements are compared to the prediction from POWHEG + PYTHIA (red line). The orange band represents its total theoretical uncertainty and the hatched red area the part of the theoretical uncertainty arising from the PDF and parton shower uncertainties. The predictions from the MC@NLO and SHERPA MC generators are also indicated by dashed and dotted-dashed lines, respectively. The SHERPA prediction is rescaled to the integrated cross section predicted by POWHEG + PYTHIA. Bottom right: The measured $W^{ \pm} Z$ differential cross section in the fiducial phase space as a function of the exclusive jet multiplicity of jets with $p_{T}>25 \mathrm{GeV}$ [3]. The measurements are compared to the prediction from SHERPA (red line), POWHEG + PYTHIA (dashed blue line) and MC@NLO (dotted-dashed violet line). 

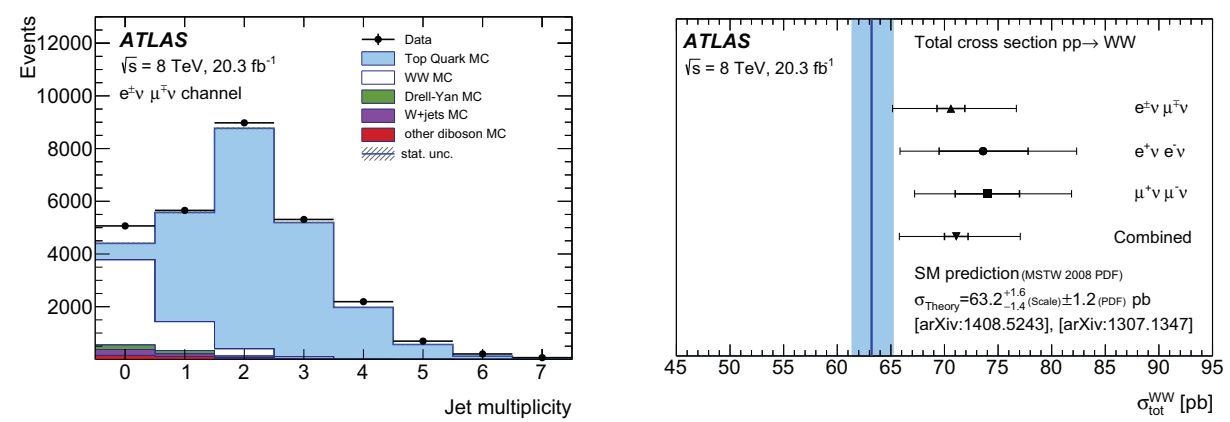

Fig. 4. - Left: Jet multiplicity distribution for $e \nu \mu \nu$ events. The points represent data and the stacked histograms are the MC predictions [4]. Only statistical uncertainties are shown. Right: The $W W$ cross sections measured at $\sqrt{s}=8 \mathrm{TeV}$ compared to the NNLO prediction [4]. The uncertainties in the measurement show the statistical as well as the total uncertainty including the luminosity uncertainty.

\section{5. - Triple and quartic gauge couplings measurements}

The non-Abelian nature of the electroweak sector of the SM predicts the selfinteraction of gauge bosons in the form of triple and quartic couplings. Searches for deviations from the SM can be parameterized in terms of anomalous Triple and Quartic Gauge Couplings (aTGC and aQGC).

To extract the aTGC, two model-independent parameterizations of possible effects beyond the SM are followed. The first makes use of an effective Lagrangian describing the $W W Z$ vertex and includes only terms that separately conserve the charge conjugation $(C)$ and parity $(P)$ quantum numbers. The deviation of the vector boson WWZ couplings
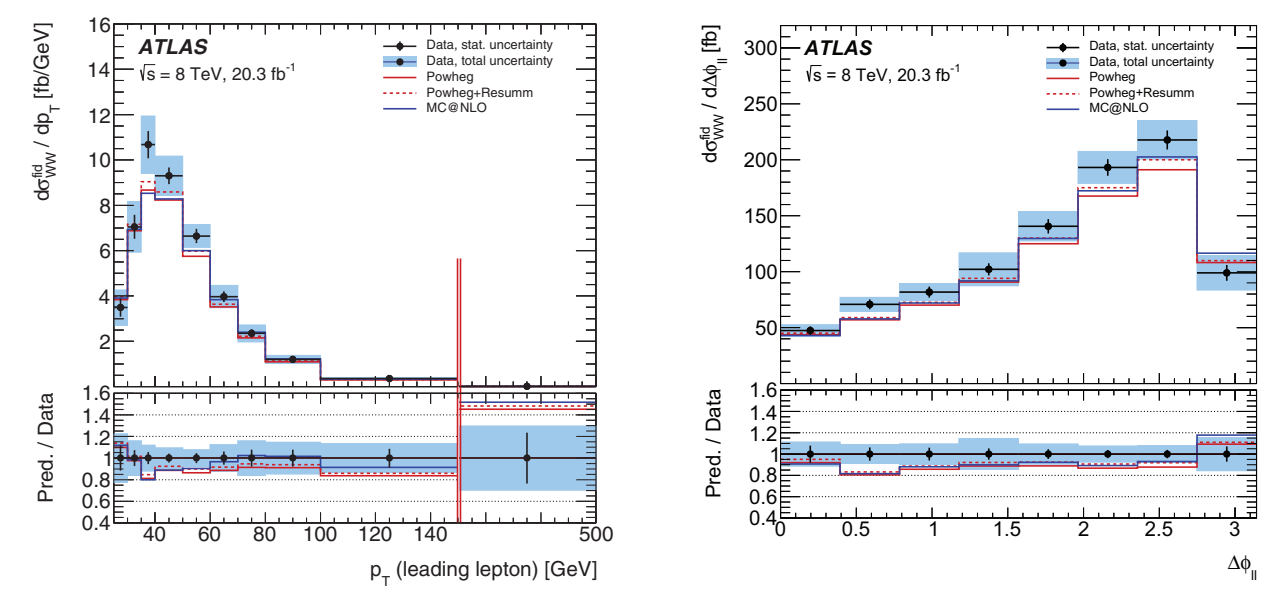

Fig. 5. - Measured unfolded differential cross sections of $W W$ production in the $e \mu$ final state for the transverse momentum of the leading lepton (left) and as the difference in azimuthal angle between the decay leptons (right) [4]. The measured cross section values are shown as markers with error bars giving the statistical uncertainty and blue bands indicating the size of the total uncertainty. The MC predictions are shown as red lines. The solid lines give the nNLO predictions that are derived using MC samples, while the dashed lines represent the approximate NNLO predictions. 

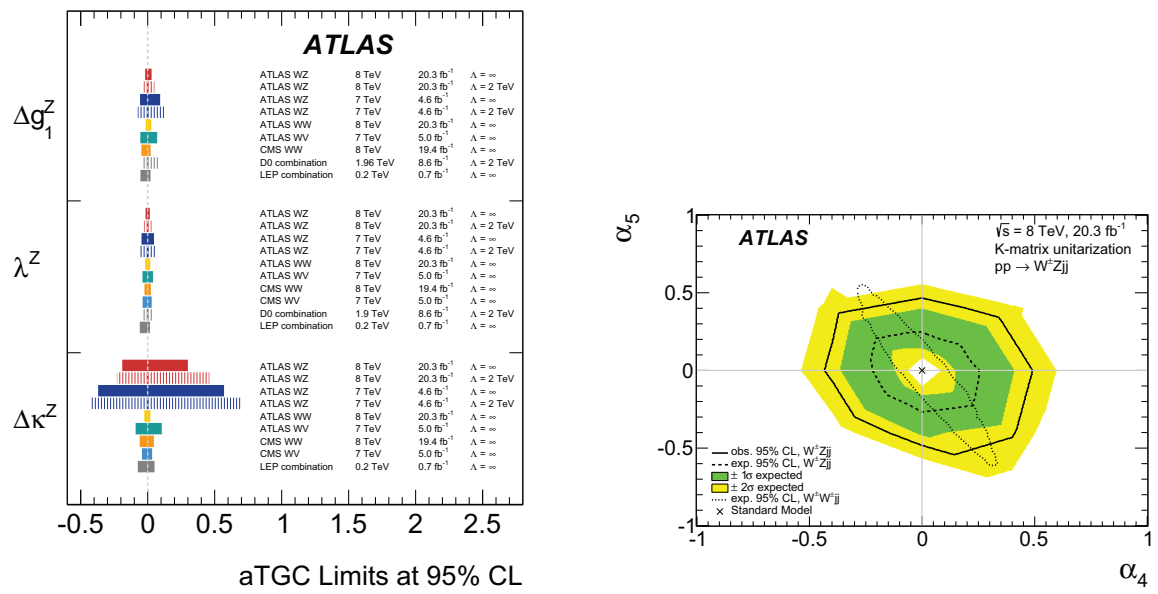

Fig. 6. - Left: Comparison of results from ATLAS, CMS, CDF, D0 and LEP experiments on limits on charged anomalous triple gauge couplings at 95\% CL [5]. Right: Expected and observed 95\% CL limit contours on aQGC parameters $\alpha_{4}$ and $\alpha_{5}$ [3]. The solid and dashed lines in the figures represent the observed and expected limits, respectively. The regions outside the black contours are excluded. The green and yellow bands correspond to the $1 \sigma$ and $2 \sigma$ uncertainty on the expected limit, respectively. The expected exclusion contour from an analysis by the ATLAS Collaboration using $W^{ \pm} W^{ \pm}+2$ jets events [6] is indicated by the dotted line.

from the SM predicted values are introduced as dimensionless anomalous couplings $\Delta k^{Z}$, $\Delta g_{1}^{Z}$ and $\lambda_{Z}$. In fig. 6(left) a summary of the limits set on the different aTGC parameters is shown along with comparisons to other experiments. Limits obtained at $\sqrt{s}=8 \mathrm{TeV}$ are usually 1.5-2.5 times better than limits at $\sqrt{s}=7 \mathrm{TeV}$. Measurements of $W W$ and $W Z$ diboson production provide complementary results and combined together they give the most stringent limits on $W W Z$ couplings.

The second parameterization is based on an effective field theory (EFT) [7] in which the particle content of the SM is not changed and the theory is extended by adding to the SM Lagrangian a linear combination of operators of mass dimension higher than four. The effective field theory allows the anomalous couplings to be reinterpreted in terms of the EFT parameters, $c_{i} / \Lambda^{2}(i=W W W, W, B)$. The dimensionless coefficients $c_{i}$ and $\Lambda$ represent the strength of the new couplings and the energy scale of new physics respectively, which provides a flexible way of parameterizing effects beyond the SM in a model-independent way. Table II presents the observed and expected one-dimensional

TABLE II. - The expected and observed 95\% confidence intervals for the EFT parameters in $W^{ \pm} Z$ (left) and $W^{+} W^{-}$(right) analyses at $\sqrt{s}=8 \mathrm{TeV}$.

\begin{tabular}{ccc}
\hline $\begin{array}{c}\text { EFT } \\
\text { coupling }\end{array}$ & $\begin{array}{c}\text { Expected } \\
{\left[\mathrm{TeV}^{-2}\right]}\end{array}$ & $\begin{array}{c}\text { Observed } \\
{\left[\mathrm{TeV}^{-2}\right]}\end{array}$ \\
\hline$c_{W} / \Lambda^{2}$ & {$[-3,7 ; 7.6]$} & {$[-4.3 ; 6.8]$} \\
$c_{B} / \Lambda^{2}$ & {$[-270 ; 180]$} & {$[-320 ; 210]$} \\
$c_{W W W} / \Lambda^{2}$ & {$[-3.9 ; 3.8]$} & {$[-3.9 ; 4.0]$} \\
\hline
\end{tabular}

\begin{tabular}{ccc}
\hline $\begin{array}{c}\text { EFT } \\
\text { coupling }\end{array}$ & $\begin{array}{c}\text { Expected } \\
{\left[\mathrm{TeV}^{-2}\right]}\end{array}$ & $\begin{array}{c}\text { Observed } \\
{\left[\mathrm{TeV}^{-2}\right]}\end{array}$ \\
\hline$c_{W} / \Lambda^{2}$ & {$[-12.58 ; 14.32]$} & {$[-5.87 ; 10.54]$} \\
$c_{B} / \Lambda^{2}$ & {$[-35.8 ; 38.4]$} & {$[-20.9 ; 26.3]$} \\
$c_{W W W} / \Lambda^{2}$ & {$[-7.62 ; 7.38]$} & {$[-4.61 ; 4.60]$} \\
\hline
\end{tabular}


intervals at $95 \% \mathrm{CL}$ on EFT coupling parameters obtained in $W^{ \pm} Z$ and $W^{+} W^{-}$analyses at $\sqrt{s}=8 \mathrm{TeV}$.

In $W^{ \pm} Z$ analysis vector boson scattering (VBS) process is also studied. This process is characterized by the presence of at least two forward jets and it is sensitive to quartic gauge couplings. Therefore, events with a $W^{ \pm} Z+2$ jets final state are used to set limits on anomalous quartic couplings in the $W Z W Z$ vertex. Deviations from the predictions of the SM are parameterized in terms of parameters $\alpha_{4}$ and $\alpha_{5}$. They are the coefficients of the two linearly independent dimension-four operators contributing to the quartic gauge couplings beyond the SM. The expected and observed two-dimensional limit contours at 95\% CL on $\alpha_{4}$ and $\alpha_{5}$ are shown in fig. 6(right). The present limit is compared to the expected limit obtained by the ATLAS Collaboration using $W^{ \pm} W^{ \pm}+2$ jets events [6]. The analysis of $W^{ \pm} Z+2$ jets events probes a domain of the $\left(\alpha_{4}, \alpha_{5}\right)$ parameter space that could not excluded by the analysis of $W^{ \pm} W^{ \pm}+2$ jets events.

\section{6. - Conclusions}

Measurements of the production cross sections of $W^{ \pm} Z, W^{ \pm} W^{ \pm}$at center of mass energy $\sqrt{s}=8 \mathrm{TeV}$ and $Z Z$ at $\sqrt{s}=13 \mathrm{TeV}$ in fully leptonic channel have been performed with the ATLAS detector. The total production cross sections are compatible with the SM expectations within uncertainties. No evidence for new physics is observed from the kinematic distributions of the diboson processes. Limits on anomalous triple and quartic gauge couplings are set. Results are also interpreted as limits on the $c_{W} / \Lambda^{2}, c_{B} / \Lambda^{2}$ and $c_{W W W} / \Lambda^{2}$ coefficients of the EFT parameterization. With an improvement by a factor of about two compared to previously existing constraints, these are the most stringent model-independent limits on WWZ anomalous couplings to date.

This work is supported by Russian MES grant RFMEFI61014X0005.

\section{REFERENCES}

[1] ATlas Collaboration, JiNST, 3 (2008) S08003.

[2] Atlas Collaboration, Phys. Rev. Lett., 116 (2016) 101801.

[3] Atlas Collaboration, Phys. Rev. D, 93 (2016) 092004.

[4] ATlas Collaboration, arXiv:1603.01702.

[5] https://atlas.web.cern.ch/Atlas/GROUPS/PHYSICS/PAPERS/STDM-2014-02/.

[6] ATlas Collaboration, Phys. Rev. Lett., 113 (2014) 141803.

[7] Degrande C. et al., Ann. Phys., 335 (2013) 21. 